\title{
Efecto del método de emplazamiento en la respuesta funcional de seis especies arbóreas de Bogotá
}

\author{
Effect of the tree-planting conditions on the functional response \\ of six arboreal species of Bogotá
}

\author{
Esteban Moreno-Barreto $^{10}$ y Kristian Rubiano ${ }^{2}$
}

Recepción: 15 de enero de 2020

Aprobación: 21 de mayo de 2020

Moreno-Barreto, E. y Rubiano, K. (2020). Efecto del método de emplazamiento en la respuesta funcional de seis especies arbóreas de Bogotá. Colombia Forestal, 23(2), 5-19.

\section{Resumen}

El incremento del arbolado urbano es una práctica cada vez más común en el mundo dada su importancia en la prestación de servicios ambientales, aunque su establecimiento se realiza bajo condiciones de espacio variables que pueden impactar su desarrollo. En este estudio se analizó la variación de rasgos funcionales de seis especies del arbolado de Bogotá en relación con distintos métodos de emplazamientos usados en la ciudad. Los resultados mostraron que no todas las especies reaccionaron de la misma forma frente a las condiciones de sellado del suelo, algunas parecen mejor adaptadas al aumento de las superficies impermeables, mientras otra parece afectarse y las demás no presentaron cambios significativos entre los distintos emplazamientos. Estos hallazgos resaltan la importancia de los rasgos funcionales de las especies, en áreas urbanas, como una herramienta para reconocer su respuesta ante las estrategias de manejo, buscando maximizar su desarrollo y la prestación de servicios ambientales.

Palabras clave: arbolado urbano, rasgos funcionales, superficies impermeables.

\begin{abstract}
The increase in urban trees is an progressively common practice around the world given its importance in the provision of different environmental services, although its establishment is carried out under variable space conditions that may impact its development. In this study, the variation of functional traits of six species of the woodland of Bogotá in relation to different methods of sites used in the city was analyzed. The results showed that not all species responded in the same way to the sealing conditions of the soils, in which some benefited from the increase in impervious surfaces, some were affected and others did not show significant changes between different locations. These findings highlight the importance of recognizing the functional component of species in urban areas as a tool for management, seeking to maximize their development and the offer of environmental services.
\end{abstract}

Keywords: urban trees, functional traits, impervious surface.

Subdirección Científica, Jardín Botánico de Bogotá José Celestino Mutis. Bogotá, Colombia. jmoreno@jbb.gov.co. Autor para correspondencia. 2 krubiano@jbb.gov.co 


\section{INTRODUCCIÓN}

Las zonas urbanas son definidas como un grupo de áreas altamente transformadas, con dominancia de una matriz muy heterogénea, la cual se compone principalmente por elementos artificiales como edificaciones e infraestructura destinada a diferentes propósitos (Godefroid y Koedam, 2007). En menor proporción, se presentan dentro de esta matriz diferentes espacios verdes que surgen como remanentes naturales de los procesos de transformación del paisaje o como zonas verdes generadas, tales como parques y jardines (Breuste et al., Niemelä y Snep, 2008). Las ciudades también se caracterizan por tener una alta concentración poblacional la cual se proyecta que incremente en los próximos años, lo que a su vez puede contribuir a un aumento de su área (Varis, 2007; Thompson y McCarthy, 2008). Estos procesos de transformación actuales y proyectados generan presiones sobre las áreas verdes y las coberturas vegetales en general, amenazando con reducir su extensión, su conectividad y su funcionalidad (Breuste et al., 2008; WiIliams et al., 2009) e impactando así la provisión de servicios ecosistémicos (Bolund y Hunhammar, 1999; Burkhard et al., 2012).

El arbolado urbano es indispensable para la provisión de servicios ecosistémicos en las ciudades, pues contribuye en la mitigación de las condiciones ambientales adversas mediante servicios como el mejoramiento de la calidad del aire, el secuestro de carbono, la regulación microclimática, los procesos de escorrentía e incrementando valores estéticos y culturales, entre otros (Bolund y Hunhammar, 1999; Tovar-Corzo, 2007; Gómez-Baggethun et al., 2013; Lovell y Taylor, 2013). Sin embargo, el arbolado urbano se ve expuesto a los desafíos ambientales que estos ecosistemas altamente transformados imponen, lo que terminan por impactar su crecimiento, vitalidad y función (Bühler et al., 2007). Lo anterior teniendo en cuenta el gran porcentaje de superficies impermeables, como andenes o calles, que normalmente son contenedores de los árboles urbanos (Just et al., 2018).
Estos contenedores son conocidos como emplazamientos y son definidos como los diferentes lugares de plantación donde se puede ubicar un árbol dentro del espacio urbano, variando en tamaño, forma y proporción de superficies impermeables (Jardín Botánico José Celestino Mutis y Dane, 2006).

Las superficies impermeables se generan a través de un proceso en el cual se elimina la cobertura presente en un área, para realizar la compactación del suelo desnudo y establecer sobre este una capa de pavimento u otro tipo de superficie impermeable (Craul, 1992). El aumento de este tipo de superficies genera fuertes presiones sobre el desarrollo de los árboles urbanos, pues se evidencian problemas como la disminución en la infiltración de agua, aumento de la compactación, disminución de la porosidad del suelo, del intercambio gaseoso y la disponibilidad de nutrientes, entre otros (Viswanathan et al., 2011; Mullaney et al., 2015; Chen et al., 2017). Todo lo cual puede afectar el desarrollo y esperanza de vida de los individuos arbóreos (Bühler et al., 2007; Mullaney et al., 2015; Just et al., 2018; Sand et al., 2018). Varios estudios han analizado el efecto de la disponibilidad del área de desarrollo, así como de la disponibilidad de agua y condiciones adecuadas del suelo, como consecuencia de la compactación y la impermeabilización del suelo en áreas urbanas alrededor del mundo. Autores como Sand et al. (2018) encontraron diferencias en el crecimiento de individuos de Tilia $\mathrm{x}$ europaea plantados en corredores arbolados y los plantados en cajas de siembra; se demostró que la salud y vitalidad del arbolado urbano se ven afectadas negativamente cuando la fracción de superficies impermeables es mayor, respaldado por otros estudios con resultados similares para diferentes especies (Grabosky y Gilman, 2004; Celestian y Martin, 2005; Ferrini y Baietto, 2007; Sanders et al., 2013; Sanders y Grabosky, 2014; Konarska et al., 2016). Estudios como el de Ow y Ghosh (2017) reportan un comportamiento opuesto, al identificar que individuos de Samanea saman y Peltophorum pterocarpum 
con espacio limitado para el desarrollo radicular crecieron más rápidamente que aquellos con espacio abierto. Por otra parte, trabajos como el de Chen et al. (2017) muestran que las especies Acer truncatum y Pinus tabuliformis presentan mayores afectaciones por condiciones de temperatura, humedad del suelo y distancia de siembra; mientras Fraxinus chinensis que mantuvo una mayor humedad del suelo presentó relaciones positivas con el crecimiento diamétrico. Por lo tanto, el crecimiento y la adaptación de los árboles al entorno urbano parece depender de cada especie, y en ese orden de ideas, no hay consenso total en la literatura respecto al efecto de las superficies impermeables en el desarrollo del arbolado urbano.

Debido a estas presiones, el desarrollo de mejores prácticas, con base técnica y científica para la selección de especies a plantar en los diferentes espacios de las ciudades, permitiría disminuir las presiones que se producen sobre el arbolado urbano y mejorar el manejo de las diferentes especies, con el fin de evitar afectaciones a la infraestructura y sobrecostos (Pretzsch et al., 2015; Núñez-Flórez et al., 2019). Un punto de partida para estos criterios de selección de las especies se da mediante la evaluación de los rasgos funcionales, como prueba de su aptitud para adaptarse a las condiciones ambientales urbanas y proveer los servicios ecosistémicos de interés (Goodnessk et al., 2016; Grote et al., 2016; Núñez-Flórez et al., 2019). Se denominan rasgos funcionales a aquellas características morfológicas, fisiológicas o fenológicas que influyen en el éxito biológico (fitness) de los organismos, permitiéndoles adaptarse a las diferentes condiciones ambientales $y$, a su vez, intervenir en los procesos de los ecosistemas (Díaz et al., 2007; Violle et al., 2007; Lavorel et al., 2011). Aunque la aplicación de este marco conceptual en el estudio de la vegetación urbana es reciente (Schwarz et al., 2017), existen algunas aproximaciones al uso de estos rasgos funcionales en relación con la prestación de diferentes servicios ecosistémicos y la capacidad de adaptación de las especies a las ciudades (e. g. Pataki et al., 2013;
Goodness et al., 2016; Grote et al., 2016; Kalusová et al., 2018; Yu et al., 2018; Núñez-Flores et al., 2019), destacando su potencial como herramienta para la determinación de criterios de selección y manejo de especies (Núñez-Flores et al., 2019).

Particularmente, en relación con la variación de rasgos funcionales como consecuencia de diferentes métodos de emplazamiento, Yu et al. (2018) encontraron que el estado de salud y la fenología de individuos de Tilia tomentosa plantados en emplazamientos predominantemente impermeables fueron afectados negativamente, a partir del análisis de rasgos foliares tales como área foliar específica, contenido foliar de agua contenido foliar de agua por área y rasgos ópticos derivados de mediciones de reflectancia y fluorescencia foliar. De manera similar, Song et al. (2015) reportan para Gingko biloba una disminución en el valor de sus rasgos fotosintéticos en las áreas con mayor porcentaje de superficies impermeables por aumentos en la temperatura hasta generar posibles procesos de fotoinhibición. Otros autores como Wang et al. (2019) hallaron de igual forma que el G. biloba es una especie que se ve afectada negativamente por el porcentaje de áreas pavimentadas, pero además por el déficit hídrico; mientras que $F$. chinensis mantuvo cambios limitados en los valores de humedad del suelo en los diferentes tratamientos, evitando así variaciones significativas en las tasas fotosintéticas.

En el presente estudio se realizó la medición de un grupo de rasgos funcionales de la hoja y el taIlo en seis de las especies arbóreas priorizadas en la ciudad de Bogotá (Colombia). Los rasgos fueron medidos en individuos establecidos en tres diferentes métodos de emplazamiento asociados a cambios en el área de desarrollo y al volumen de suelo y de agua disponible. A partir de esto, nuestros objetivos fueron: examinar el efecto de los tres métodos de emplazamiento sobre la expresión de los rasgos funcionales de los árboles y comparar la variación de los individuos dependiendo de la especie evaluada, con el fin de proveer información que aporte criterios para la selección de especies a plantar en estos tres tipos de emplazamiento. 


\section{MATERIALES Y MÉTODOS}

\section{Área de estudio}

Los muestreos se realizaron en el área urbana de la ciudad de Bogotá (Colombia), tanto en espacios verdes como en espacios predominantemente impermeables, en los que se encontraron individuos de las especies objeto de estudio en los tipos de emplazamiento deseados. Bogotá se ubica a una altitud promedio de $2630 \mathrm{~m}$ y se desarrolla con las condiciones ambientales de un bosque andino (Barrera-Cataño et al., 2010), donde se registra una precipitación media anual de $1000 \mathrm{~mm}$ y una temperatura promedio que varía entre los 12 y $15^{\circ} \mathrm{C}$. Tiene una población urbana de aproximadamente 8 millones de habitantes y una superficie de alrededor de 380 km² (Cristancho y Triana, 2018).

\section{Selección de especies y tipos de emplazamiento}

Para cuantificar los cambios en los rasgos de las especies arbóreas bajo tres diferentes tipos de emplazamiento distribuidos en la ciudad se seleccionaron seis especies priorizadas por el Jardín Botánico de Bogotá para la investigación y plantación: Caucho sabanero (Ficus americana subsp. andicola (Standl.) C.C.Berg), Falso pimiento (Schinus areira L.), Guayacán de Manizales (Lafoensia acuminata (Ruiz \& Pav.) DC.), Jazmín del Cabo (Pittosporum undulatum Vent.), Liquidámbar (Liquidambar styraciflua L.) y Roble (Quercus humboldtii Bonpl.). Para cada una de las especies se realizó un muestreo de 30 individuos por cada tipo de emplazamiento, contabilizando 90 individuos por especie y un total de 540 individuos muestreados. Los individuos muestreados se seleccionaron usando como referencia el Sistema de Información Geográfica para la Gestión del Arbolado Urbano (Sigau), el cual consiste en una base de datos que contiene información del censo del arbolado de Bogotá, que incluye datos como especie, tipo de emplazamiento y coordenadas geográficas de cada individuo (Jardín
Botánico José Celestino Mutis y Dane, 2006). Estos datos permitieron filtrar las zonas de la ciudad con presencia de individuos de interés para la investigación, es decir, de las especies y en los emplazamientos de interés. En estas áreas se muestrearon individuos con un DAP mayor a $10 \mathrm{~cm}$ que cumplieron con los lineamientos planteados por los protocolos para la medición de rasgos funcionales (Cornelissen et al., 2003; Pérez-Harguindeguy et al., 2013; Salgado-Negret, 2015).

Los tipos de emplazamiento evaluados incluyeron (a) pozos de plantación: árboles individuales plantados en pequeños espacios rodeados totalmente por una superficie impermeable; (b) corredores arbolados: grupos de árboles plantados en franjas de suelo (como separadores viales o andenes arbolados) que siguen estando rodeadas por un alto porcentaje de superficies impermeables pero no totalmente; y (c) parques urbanos o espacio ilimitado: árboles plantados dentro de amplios espacios verdes con menor porcentaje de superficies impermeables a su alrededor (figura 1).

\section{Medición de rasgos funcionales}

Los muestreos se realizaron entre los meses de abril y julio del 2019. A cada uno de los árboles seleccionados se le midió la altura $(\mathrm{H}, \mathrm{m})$, las longitudes $X$ y $Y$ de la proyección ortogonal de la copa para estimar su área $\left(\mathrm{AC}, \mathrm{m}^{2}\right)$ y el diámetro a la altura del pecho (DAP, cm), usando un decámetro y un hipsómetro digital Nikon Forestry Pro $®$. Para los individuos que presentaban bifurcaciones por debajo de $1.3 \mathrm{~m}$ de altura, el valor de su DAP fue calculado mediante la ecuación del diámetro cuadrático promedio (Diéguez et al., 2003). Además, se colectó una rama expuesta al Sol, de la cual se obtuvo una muestra de madera y cinco hojas en buen estado sanitario, siguiendo los protocolos para la medición de rasgos funcionales (Cornelissen et al., 2003; Pérez-Harguindeguy et al., 2013; Salgado-Negret 2015). Las muestras fueron transportadas al laboratorio en bolsas de cierre hermético, envueltas en papel húmedo para 


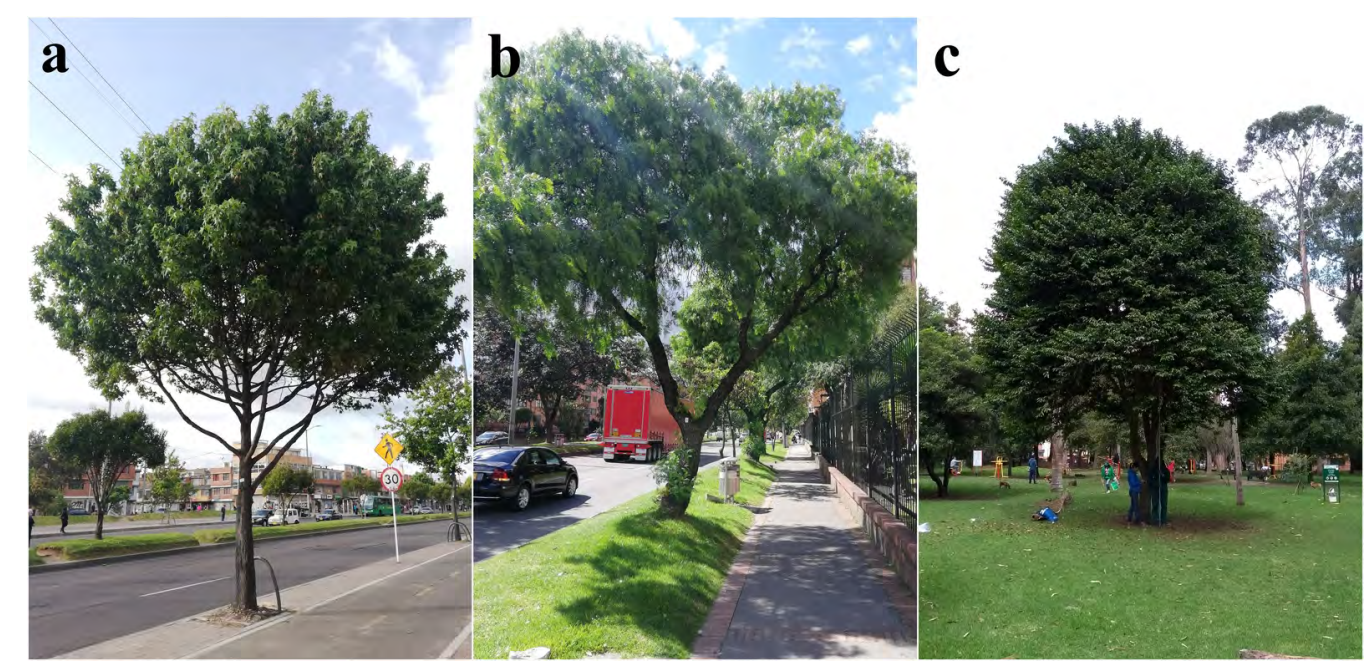

Figura 1. Métodos de emplazamiento evaluados para las seis especies objeto de estudio: a) Pozos de plantación, b) corredores arbolados y, c) parques.

evitar su deshidratación y fueron almacenadas en nevera hasta su procesamiento.

Para determinar la densidad básica de la madera (DB, g. $\mathrm{cm}^{-3}$ ) las muestras se descortezaron y se rehidrataron para obtener su volumen fresco a partir del método de desplazamiento de agua. Después se ingresaron al horno de secado a una temperatura de $70^{\circ} \mathrm{C}$ por 72 horas y, posterior a esto, se determinó el peso seco de las muestras. Además de DB, se midió la densidad anhidra $\left(\mathrm{DO}, \mathrm{g} . \mathrm{cm}^{-3}\right)$, reemplazando el volumen fresco de las muestras por su valor secado al horno. Para determinar los valores de contenido máximo de agua en el tallo (CHMAX, \%) se siguió la metodología propuesta por Simpson (1993). Las muestras foliares se fotografiaron incluyendo el pecíolo, con una escala conocida sobre un fondo blanco y cubiertas por un vidrio antirreflejo. Posteriormente, se estimó su área foliar $\left(\mathrm{AF}, \mathrm{mm}^{2}\right)$ mediante el software ImageJ $囚$ (Schneider, Rasband y Eliceiri, 2012). Adicional a esto, se midió el peso fresco de cada hoja y el peso seco luego de llevar al horno de secado a una temperatura de $60^{\circ} \mathrm{C}$ por 72 horas. A partir de estos valores se calcularon el área foliar específica (AFE, $\mathrm{cm}^{2} \cdot \mathrm{g}^{-1}$ ), el contenido foliar de materia seca (CFMS, mg. ${ }^{-1}$ ) y el contenido de agua foliar (CAF, \%) (Cornelissen et al., 2003; Pérez-Harguindeguy et al., 2013; Salgado-Negret 2015). Los valores de los rasgos foliares se promediaron para cada individuo a partir de las cinco mediciones.

Los rasgos funcionales seleccionados corresponden a rasgos blandos, los cuales se caracterizan por su facilidad de medición y por no generar daños importantes a los individuos, a la vez que aportan información pertinente para el estudio funcional de la vegetación. Se seleccionaron ya que, al tratarse de individuos localizados en área urbana, no es adecuada la implementación de metodologías destructivas que pongan en riesgo su salud y vitalidad, debido a que hacen parte del patrimonio público de la ciudad. De otro lado, su selección se basó en los antecedentes encontrados en la literatura y en los mecanismos que relacionan estos rasgos con la respuesta funcional a las características de los diferentes emplazamientos analizados. El estrés hídrico generado por el aumento de superficies impermeables puede inducir cambios en la morfología foliar manifestados en menor AF y en reducción de AFE y CAF (Farooq et al., 2009; Xu et al., 2009). A su vez, puede afectar rasgos hidráulicos como la densidad de la madera y el contenido de humedad máximo del tallo, los cuales se relacionan con la conductividad hidráulica y el suministro hídrico a las hojas (Chave et al., 2009; Salgado-Negret, 2015). La alteración del suelo y en consecuencia la modificación de la disponibilidad 
y ciclaje de nutrientes puede afectar rasgos como CFMS y AFE (Wright et al., 2004).

\section{Análisis de datos}

Para evitar los efectos de la autocorrelación en los análisis se realizó una prueba de Pearson con el fin de determinar los rasgos altamente correlacionados y no incluir aquellos rasgos redundantes en los análisis posteriores. De acuerdo con la prueba, los rasgos que se mantuvieron para los análisis posteriores fueron AF, AFE, CFMS, DB y DO. Para cada uno de estos rasgos se realizó un Anova de transformación alineada de rangos (Aligned Ranks Transform Anova) para dos factores (Wobbrock et al., 2011), usando como factores el tipo de emplazamiento y la especie, y teniendo en cuenta la interacción entre ellos. Luego se realizaron las respectivas pruebas post hoc de Tukey para identificar diferencias puntuales entre niveles y sus interacciones. Los análisis se realizaron utilizando las librerías ARTool (Kay y Wobbrock, 2019) y Emmeans (Lenth, 2019), respectivamente, del paquete estadístico R versión 3.5.3 (R Core Team, 2019).

\section{RESULTADOS}

\section{Relación de los rasgos funcionales con las superficies impermeables}

Los resultados de la prueba mostraron que los rasgos $\mathrm{AF}, \mathrm{AFE}, \mathrm{CFMS}, \mathrm{DB}$ y $\mathrm{DO}$ son los que presentan un menor grado de correlación. Por el contrario, CAF y CHMAX se correlacionaron entre sí y con CFMS, DO y DB. Además, se encontró que las variables $\mathrm{H}$ y DAP no mostraron alguna relación significativa con los rasgos funcionales, a excepción de la relación entre DAP-DO $(\rho=-0.35$, $\mathrm{p}<0.001$; figura 2).

Los resultados del Anova muestran que el AF varía significativamente entre los diferentes métodos de emplazamiento (tabla 1), registrando los valores más altos en el método de emplazamiento con mayor porcentaje de área verde (i. e., parques) respecto a los otros dos métodos (tabla 2). El caso de DO fue similar al de AF, con sus valores más altos en los parques urbanos, respecto a los corredores y pozos ( $\mathrm{F}=17.47 ; \mathrm{p}<0.01)$. Los rasgos $\mathrm{AFE}(\mathrm{F}=28.49 ; \mathrm{p}<0.001)$ y $\mathrm{CFMS}(\mathrm{F}=5.56$;

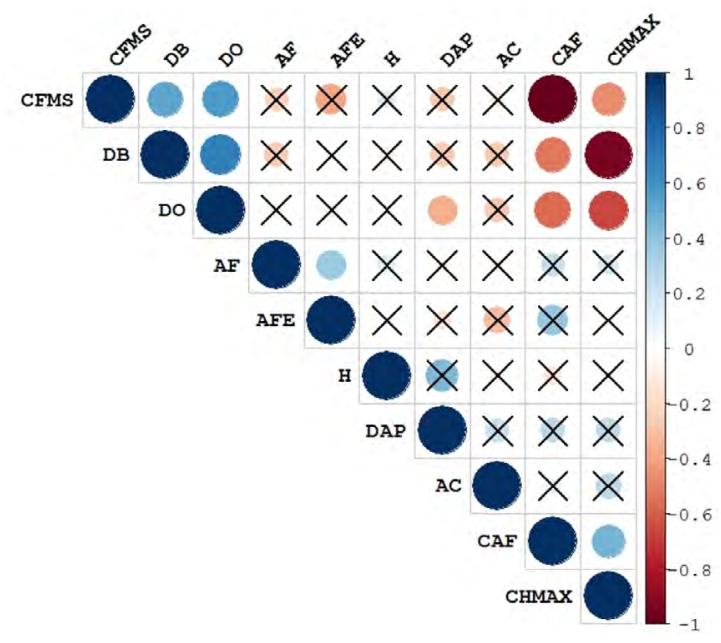

Figura 2. Correlaciones de Pearson entre rasgos funcionales y variables morfológicas. Círculos más grandes representan mayor nivel de significancia ( $p<0.05$ ). El color indica la dirección y la fuerza de la correlación: rojo correlación negativa y azul correlación positiva. Las cruces negras indican correlaciones estadísticamente no significativas. AF (área foliar), AFE (área foliar específica), AC (área de copa), CAF (contenido de agua foliar), CFMS (contenido foliar de materia seca), CHMAX (contenido de humedad máximo del tallo), DAP (diámetro a la altura del pecho), DB (densidad básica de la madera), DO (Densidad anhidra de la madera), H (altura del árbol). 
$\mathrm{p}<0.001)$ presentaron un comportamiento contrario, donde los valores más altos se reportaron en los emplazamientos de pozos de plantación y corredores arbolados, en relación con los parques urbanos (tabla 2). El rasgo DB no presentó variación entre los tres métodos de emplazamiento evalua$\operatorname{dos}(\mathrm{F}=0.60 ; \mathrm{p}=0.548)$.

Para el caso de las diferencias entre el grupo de especies, como se esperaba por resultados obtenidos en otros estudios (Ocampo, 2019), para todos los rasgos se presentaron diferencias significativas entre las seis especies (tabla 1). En todos los casos, al realizar las pruebas post hoc, se observa en promedio la conformación de cinco grupos significativos (tabla 2), lo que permite analizar el impacto de los diferentes métodos de emplazamiento en el desarrollo de estas especies con distintas estrategias funcionales.

\section{Interacción rasgos funcionales y métodos de emplazamiento}

Se encontró que la interacción entre las especies y los métodos de emplazamiento evaluados respecto a cada rasgo funcional fue estadísticamente significativa para todos los casos (tabla 1). Al

Tabla 1. Resultados de la prueba de Anova para cada uno de los rasgos funcionales analizados. Valores en negrilla representan diferencias a un nivel de significancia de 0.05

\begin{tabular}{lccc}
\hline & GL & F & p-valor \\
\hline AFE & & & \\
Especie & 5 & 172.57 & $<\mathbf{0 . 0 0 1}$ \\
Emplazamiento & 2 & 28.49 & $<\mathbf{0 . 0 0 1}$ \\
Especie*Emplazamiento & 10 & 5.16 & $<\mathbf{0 . 0 0 1}$ \\
AF & & & \\
Especie & 5 & 94.54 & $<\mathbf{0 . 0 0 1}$ \\
Emplazamiento & 2 & 4.99 & $<\mathbf{0 . 0 0 7}$ \\
Especie*Emplazamiento & 10 & 7.43 & $<\mathbf{0 . 0 0 1}$ \\
CFMS & & & \\
Especie & 5 & 207.45 & $<\mathbf{0 . 0 0 1}$ \\
Emplazamiento & 2 & 5.56 & $<\mathbf{0 . 0 0 4}$ \\
Especie* Emplazamiento & 10 & 5.71 & $<\mathbf{0 . 0 0 1}$ \\
DB & & & \\
Especie & 5 & 143.05 & $<\mathbf{0 . 0 0 1}$ \\
Emplazamiento & 2 & 0.60 & $\mathbf{0 . 5 4 8 1}$ \\
Especie* Emplazamiento & 10 & 4.86 & $<\mathbf{0 . 0 0 1}$ \\
DO & & & \\
Especie & 5 & 133.25 & $<\mathbf{0 . 0 0 1}$ \\
Emplazamiento & 2 & 17.47 & $<\mathbf{0 . 0 0 1}$ \\
Especie* Emplazamiento & 10 & 3.60 & $<\mathbf{0 . 0 0 1}$ \\
\hline
\end{tabular}

Tabla 2. Valores de la mediana y resultados de la prueba post hoc de Tukey para cada factor. Letras diferentes representan diferencias a un nivel de significancia de 0.05

\begin{tabular}{|c|c|c|c|c|c|c|c|c|c|c|c|}
\hline \multirow{2}{*}{ Factor } & \multirow{2}{*}{ Niveles } & \multicolumn{2}{|l|}{ AF } & \multicolumn{2}{|l|}{ AFE } & \multicolumn{2}{|l|}{ CFMS } & \multicolumn{2}{|l|}{ DB } & \multicolumn{2}{|l|}{ DO } \\
\hline & & Mediana & & Mediana & & Mediana & & Mediana & & Mediana & \\
\hline & Pozo & 4251.9 & $a b$ & 87.6 & $\mathrm{~b}$ & 417.6 & $\mathrm{~b}$ & 0.52 & $\mathrm{a}$ & 0.64 & $\mathrm{a}$ \\
\hline Emplazamiento & Corredor & 4037.8 & $\mathrm{a}$ & 88.1 & $\mathrm{~b}$ & 421.7 & b & 0.52 & $\mathrm{a}$ & 0.63 & $\mathrm{a}$ \\
\hline & Parque & 4205.0 & $\mathrm{~b}$ & 77.3 & $\mathrm{a}$ & 386.7 & $\mathrm{a}$ & 0.52 & $\mathrm{a}$ & 0.67 & $b$ \\
\hline & F. americana subsp. andicola & 3867.2 & $\mathrm{a}$ & 60.5 & $\mathrm{a}$ & 377.6 & $b$ & 0.42 & a & 0.53 & $\mathrm{a}$ \\
\hline & L. acuminata & 4140.9 & $\mathrm{C}$ & 90.8 & d & 398.0 & $\mathrm{a}$ & 0.54 & b & 0.65 & $b$ \\
\hline Fsne & L. styraciflua & 5876.9 & $\mathrm{C}$ & 112.4 & $f$ & 408.1 & C & 0.50 & d & 0.62 & $b$ \\
\hline Especie & P. undulatum & 2399.3 & $\mathrm{~b}$ & 70.6 & $\mathrm{~b}$ & 462.0 & d & 0.60 & e & 0.68 & C \\
\hline & Q. humboldtii & 4315.4 & $d$ & 77.9 & C & 481.8 & C & 0.53 & C & 0.77 & d \\
\hline & S. areira & 4538.5 & $\mathrm{C}$ & 102.0 & $\mathrm{e}$ & 337.6 & $\mathrm{e}$ & 0.48 & d & 0.62 & $\mathrm{e}$ \\
\hline
\end{tabular}


determinar los grupos significativos, se encontró que $L$. acuminata es la especie que presentó una mayor variación en sus rasgos entre los métodos de emplazamiento (AF, CFMS, DB y DO), seguido por $S$. areira (AFE, CFMS y densidad de la madera) y $Q$. humboldtii (AF y DB), aunque la primera especie presentó un comportamiento opuesto al de las dos últimas. El resto de las especies evaluadas (F. americana subsp. andicola, L. styraciflua y $P$. undulatum) no presentaron variaciones significativas entre los diferentes métodos de emplazamiento en ninguno de los rasgos evaluados (figura 3).
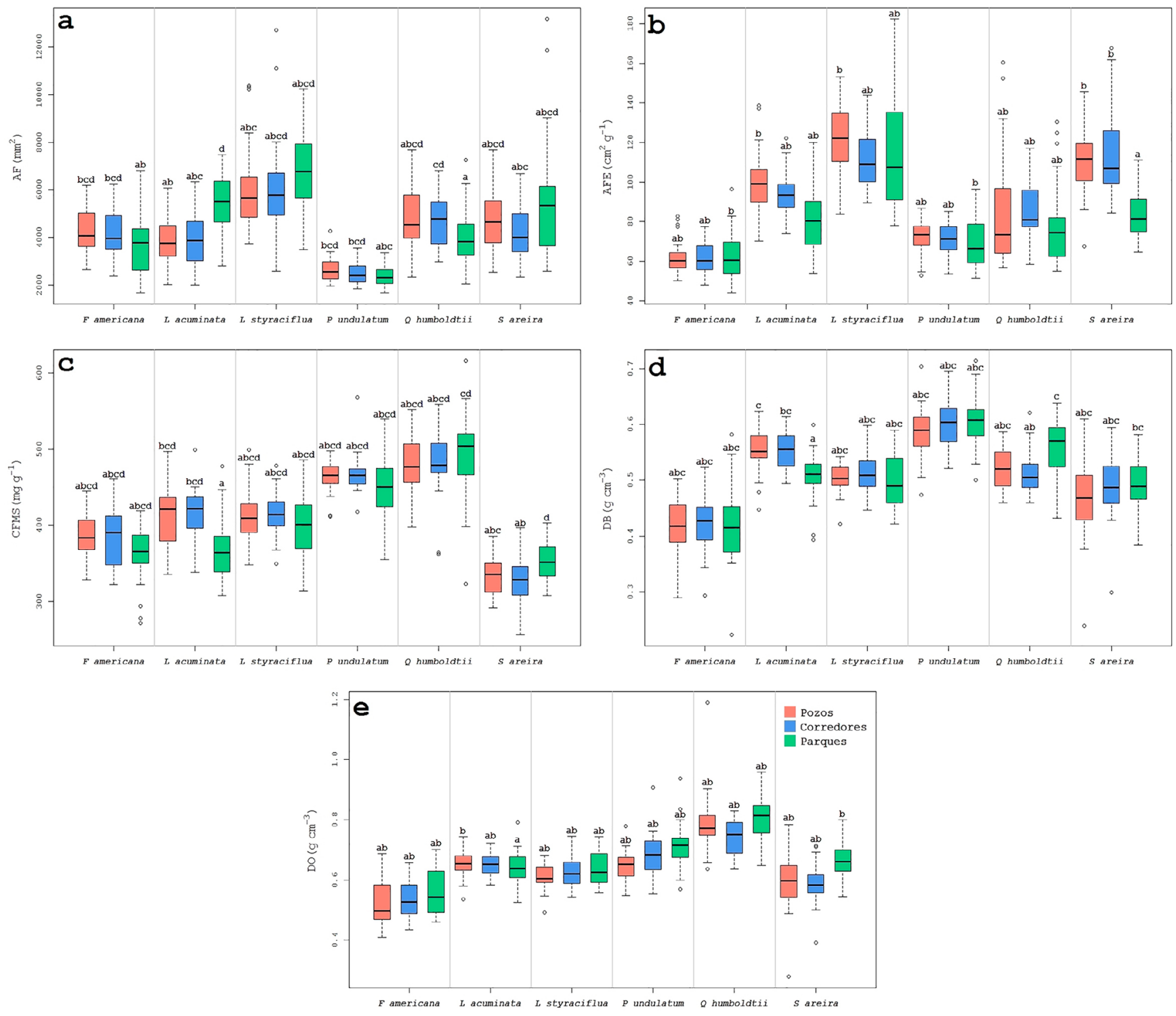

Figura 3. Diagramas de boxplots de las interacciones entre especies y métodos de emplazamiento para los diferentes rasgos funcionales. a) Área foliar (AF), b) área foliar específica (AFE), c) contenido foliar de materia seca (CFMS), d) densidad básica de la madera (DB) y e) densidad anhidra de la madera (DO). Letras no compartidas representan diferencias a un nivel de significación de 0.05 . 


\section{DISCUSIÓN}

En general, los resultados indican que todos los rasgos analizados variaron significativamente entre especies. Esto se esperaba teniendo en cuenta las diferentes estrategias funcionales que presentan las especies, como resultado de su historia filogenética y las adaptaciones a las condiciones ambientales de sus lugares de origen (Wright et al., 2004; Freschet et al., 2010). Sin embargo, estas diferencias son las que permiten interpretar como más o menos apta a una especie para su desarrollo y funcionamiento en las condiciones de estrés urbano, incluyendo los diferentes grados de impermeabilización de los suelos (Bühler et al., 2007; Just et al., 2018). Por esta razón, este tipo de resultados son un importante aporte para la selección de especies y el manejo del arbolado urbano, con el fin de maximizar la provisión de servicios ecosistémicos (Calfaprieta et al., 2015; Núñez-Flórez et al., 2019). Las diferencias identificadas en la expresión de los rasgos funcionales entre las especies evaluadas concuerdan con otros estudios que analizaron el comportamiento de otro grupo de rasgos para estas mismas especies en la ciudad de Bogotá y analizaron su variación intraespecífica frente a condiciones del medio urbano (Moreno-Barreto y Rubiano, 2018; Ocampo, 2019).

En cuanto a los emplazamientos, se identificaron diferencias para todos los rasgos, con excepción de DB que no fue significativo (tabla 1). Al analizar los resultados de los análisis post hoc se observa que, para las especies en conjunto, los pozos y corredores tendieron a agruparse y a diferenciarse de los parques (tabla 2). Esto podría reflejar un efecto negativo de las superficies impermeables sobre los rasgos analizados y coincide con la mayoría de la evidencia aportada por los estudios que analizan este tipo de efectos (e. g. Grabosky y Gilman, 2004; Celestian y Martin, 2005; Ferrini y Baietto, 2007; Sanders et al., 2013; Sanders y Grabosky, 2014; Konarska et al., 2016). Estas diferencias se deberían a las modificaciones que generan las superficies impermeables sobre las condiciones de desarrollo de los árboles, particularmente reflejadas en el espacio disponible y las características del suelo, que se asemejan más a las condiciones naturales en los parques (Viswanathan et al., 2011; Chen et al., 2016). Yu et al. (2018) identificaron diferencias entre pozos y corredores respecto a las áreas sin superficies impermeables, donde los individuos del último tipo de emplazamiento presentaron valores más altos de AFE, en concordancia con el comportamiento de algunas de las especies aquí evaluadas, posiblemente con estrategias funcionales similares.

$\mathrm{Al}$ analizar las interacciones entre las especies y los tipos de emplazamiento se encontró que la mitad de las especies presentó algún tipo de variación significativa en relación con el área de crecimiento radicular, y que estas a su vez se distinguieron de acuerdo con la forma en la que se presentaron las variaciones. L. acuminata tuvo un comportamiento similar al esperado, donde se observó una afectación sobre el crecimiento de la especie en relación con el aumento de las superficies impermeables; con una disminución de AF mientras rasgos como CFMS, DB y DO se vieron incrementados. Yu et al. (2018) también reportan afectaciones sobre rasgos foliares de Tilia tomentosa como AFE y CAF (opuesto a CFMS) en relación con el aumento en áreas impermeables, donde las especies buscan generar estructuras más resistentes, dados a los problemas que acarrea la presencia de este tipo de superficies para el desarrollo vegetal como el déficit hídrico, la limitación del crecimiento radicular, reducción de tasas fotosintéticas, el aumento de la temperatura o la disminución de la humedad del suelo, entre otras (Viswanathan et al., 2011; Chen et al., 2017; McClung e Ibáñez, 2018; Sand et al., 2018; Wang et al., 2019, Yan et al., 2019). Las especies Q. humboldtii y $S$. areira, por otro lado, parecen mejor adaptadas al aumento de las superficies impermeables, donde la primera presentó un aumento en su AF con una disminución en DB, mientras que $S$. areira tuvo un aumento en los valores de AFE con una disminución en CFMS y la densidad de madera. Autores como Vallet et al. (2010) 
sugieren que dentro de las áreas urbanas se destaca la presencia de aquellas especies preferentes por suelos básicos que se favorecen por procesos como la deposición de polvo de cemento y un aumento en la fertilidad del suelo como consecuencia de la combustión y deposición de nitrógeno, entre otros, en donde se resalta la presencia de una gama de especies con valores altos para AF y AFE (Thompson y McCarthy, 2008); mientras Chen et al. (2016) describen una tasa de brotación temprana en los árboles urbanos, que se ve beneficiada por un aumento de la temperatura generado por mayores porcentajes de cobertura impermeable. Carreras et al. (1996) reportan aumentos en los valores de AFE de Ligustrum lucidum en áreas con altos niveles de tráfico, aunque no sucede lo mismo con Ligustrum lucidum f. tricolor, por lo que la variación de este rasgo en las especies estudiadas podría ser una estrategia para sobrevivir al medio urbano, debido a la relación positiva que tiene con las tasas fotosintéticas y la preferencia de especies con esta característica por áreas con altas concentraciones de nutrientes (Wright et al., 2004, Ordóñez et al., 2009).

El resto de las especies ( $F$. americana subsp. andicola, L. styraciflua y $P$. undulatum) no presentaron variaciones significativas en ninguno de los rasgos funcionales medidos en individuos establecidos en diferentes tipos de emplazamientos (figura 3). Estudios como el de Wang et al. (2019) han encontrado resultados similares al evaluar otros rasgos, en los que determinaron que las tasas fotosintéticas de $F$. chinensis no se vieron afectadas por el aumento de las áreas impermeabilizadas debido, posiblemente, a características propias de las especies como condiciones de raíz que permiten mejorar la aireación del suelo en el que se establece, hasta llegarse a reportar tasas de crecimiento más altas que otras especies bajo las mismas condiciones (Chen et al., 2017). De igual forma, McClung e Ibáñez (2018) encontraron que el crecimiento de Carya ovata no se vio afectado por el aumento de las superficies urbanas ni el efecto de la sequía en comparación con otras dos especies evaluadas; al igual que Carreras et al. (1996), quienes no encontraron variaciones para las características fisiológicas y funcionales de Ligustrum lucidum f. tricolor en relación con diferentes grados de tráfico e industrialización. Se asume que la ausencia de variación de estas tres especies entre los distintos métodos de emplazamiento puede estar asociada a tres posibles causas: una alta resistencia a las condiciones adversas que se presentan en las ciudades, a través de distintas estrategias como algunas características propias de cada especie (e. g. Wang et al., 2019); una baja variación intraespecífica que puede estar relacionada con una baja capacidad de adaptación de las especies frente al medio urbano (Albert et al., 2010; Salgado-Negret, 2015), que podría verse reflejado en una afectación de la vitalidad de estas especies con los fuertes cambios que se esperan en el futuro para los ecosistemas urbanos (Farrell et al., 2015); o que posiblemente los rasgos evaluados no fueron los adecuados para determinar variaciones en estas especies, por lo que el uso de otro grupo de rasgos como los atributos fotosintéticos o radiculares podría mostrar cambios en las especies frente a las condiciones del medio urbano.

\section{CONCLUSIONES}

Aunque en los últimos años la investigación sobre las coberturas vegetales presentes en las ciudades se ha incrementado de forma sustancial debido a las condiciones futuras que se prevé se presentarán en las zonas urbanizadas, aún se tiene un vacío en el conocimiento funcional de las especies sembradas en estas áreas bajo la influencia de múltiples estresores, lo que podría mejorar las prácticas de manejo de los árboles urbanos y, con ello, la prestación de diferentes servicios. Este estudio investigó cómo distintos métodos de emplazamiento en las áreas urbanas afectan el estado de desarrollo de los árboles al medir un conjunto de rasgos funcionales en seis especies arbóreas comunes dentro de la ciudad de Bogotá. Los resultados mostraron 
que tres de las seis especies de árboles estaban influenciadas por la variación en el porcentaje de superficie impermeable, donde dos de estas parecen estar mejor adaptadas a las condiciones del medio urbano mientras los árboles de $L$. acuminata parecen ser afectados por el aumento del área pavimentada. Esto implica que las especies pueden reaccionar de forma distinta a las mismas condiciones ambientales de acuerdo con las diferentes estrategias funcionales que cada una de ellas posee, beneficiándose algunas, perjudicando a otras o sin generar algún tipo de alteración. Por esto se hace necesario ampliar el conocimiento funcional de las especies que se encuentran presentes dentro de las ciudades como factor clave a ser considerado en la planeación y manejo del arbolado urbano. Recomendamos evitar la plantación de L. acuminata en áreas con poco espacio para el crecimiento radicular debido a que fue la única especie que vio afectada la expresión de sus rasgos por el aumento de las superficies impermeables. Además, evaluar el efecto de otras variables que actúan dentro del espacio urbano como posibles estresores de los árboles (e. g. tráfico urbano, captación de contaminantes o gradientes de polución), junto con el uso de otro grupo de rasgos funcionales que aporten información valiosa a la evaluación del desarrollo de las plantas urbanas como los rasgos fotosintéticos, de tipo radicular o asociados a la arquitectura de la copa.

\section{AGRADECIMIENTOS}

Esta investigación fue realizada en la línea de Interacciones y Coberturas Vegetales Urbanas de la subdirección científica, bajo los contratos de prestación de servicios 063-2019 y 474-2019 del Jardín Botánico de Bogotá José Celestino Mutis. Los autores expresan su agradecimiento a los operarios Laura Navarrete y Milton Gómez por su apoyo en las actividades de campo y en el procesamiento del material vegetal en los laboratorios del Jardín Botánico José Celestino Mutis.

\section{CONFLICTO DE INTERESES}

Los autores declaran no tener conflicto de intereses.

\section{CONTRIBUCIÓN POR AUTOR}

E.M.B. y K.R. planearon y diseñaron la investigación, coordinaron y llevaron a cabo el trabajo en campo y laboratorio, analizaron los datos y redactaron el manuscrito. Ambos autores contribuyeron a la discusión y comentaron los borradores.

\section{REFERENCIAS}

Albert, C. H., Thuiller, W., Yoccoz, N. G., Soudant, A., Boucher, F., Saccone, P. y Lavorel, S. (2010). Intraspecific functional variability: Extent, structure and sources of variation. Journal of Ecology, 98, 604-613.

https://doi.org/10.1111/j.1365-2745.2010.01651.x

Barrera-Cataño, J., Contreras-Rodríguez, S., Garzón-Yepes, N., Moreno-Cárdenas, A. y Montoya-Villarreal, S. (2010). Manual para la restauración ecológica de los ecosistemas disturbados del Distrito Capital. Bogotá: Secretaría Distrital de Ambiente; Pontificia Universidad Javeriana.

Bolund, P. y Hunhammar, S. (1999). Ecosystem services in urban areas. Ecological Economics, 29(2), 293-301.

https://doi.org/10.1016/s0921-8009(99)00013-0

Breuste, J., Niemelä, J. y Snep, R. P. H. (2008). Applying landscape ecological principles in urban environments. Landscape Ecology, 23(10), 1139-1142. https://doi.org/10.1007/s10980-008-9273-0

Bühler, O., Kristoffersen, P. y Larsen, S. U. (2007). Growth of street trees in Copenhagen with emphasis on the effect of different establishment concepts. Arboriculture \& Urban Forestry, 33(5), 330-337.

Burkhard, B., Kroll, F., Nedkov, S. y Müller, F. (2012). Mapping ecosystem service supply, demand and budgets. Ecological Indicators, 21, 17-29. https://doi.org/10.1016/j.ecolind.2011.06.019 
Calfapietra, C., Peñuelas, J. y Niinemets, Ü. (2015). Urban plant physiology: adaptation-mitigation strategies under permanent stress. Trends in Plant Science, 20(2), 72-75.

https://doi.org/10.1016/j.tplants.2014.11.001

Carreras, H. A., Cañas, M. S. y Pignata, M. L. (1996). Differences in responses to urban air pollutants by Ligustrum lucidum Ait. and Ligustrum lucidum Ait. f. tricolor (Rehd.) Rehd. Environmental Pollution, 93(2), 211-218.

https://doi.org/10.1016/0269-7491(96)00014-0

Celestian, S. y Martin, C. (2005). Effects of parking lot location on size and physiology of four southwestern US landscape trees. Journal of Arboriculture, 31(4), 191-197.

Chave, J., Coomes, D., Jansen, S., Lewis, S. L., Swenson, N. G. y Zanne, A. E. (2009). Towards a worldwide wood economics spectrum. Ecology Letters, 12(4), 351-366.

https://doi.org/10.1111/j.1461-0248.2009.01285.x

Chen, Y., Wang, X., Jiang, B., Yang, N. y Li, L. (2016). Pavement induced soil warming accelerates leaf budburst of ash trees. Urban Forestry \& Urban Greening, 16, 36-42.

https://doi.org/10.1016/j.ufug.2016.01.014

Chen, Y., Wang, X., Jiang, B., Wen, Z., Yang, N. y Li, L. (2017). Tree survival and growth are impacted by increased surface temperature on paved land. Landscape \& Urban Planning, 162, 68-79.

https://doi.org/10.1016/j.landurbplan.2017.02.001

Cornelissen, J. H. C., Lavorel, S., Garnier, E., Díaz, S., Buchmann, N., Gurvich, D. E., Reich, P. B., ter Steege, H., Morgan, H. D., van der Heijden, M. G. A., Pausas, J. G. y Poorter, H. (2003). A handbook of protocols for standardised and easy measurement of plant functional traits worldwide. Australian Journal of Botany, 51(4), 335-380.

https://doi.org/10.1071/bt02124

Craul, P. J. (1992). Urban Soil in Landscape Design. Nueva York: Wiley.

Cristancho, C. y Triana, E. (2018). Análisis demográfico y proyecciones poblacionales de Bogotá. Bogotá: Secretaría Distrital de Planeación.

http://www.sdp.gov.co/sites/default/files/demografia_proyecciones_2017_0_0.pdf
Díaz, S., Lavorel, S., de Bello, F., Quétier, F., Grigulis, K. y Robson, T. M. (2007). Incorporating plant functional diversity effects in ecosystem service assessments. PNAS, 104(52), 20684-20689.

https://doi.org/10.1073/pnas.0704716104

Diéguez, U., Barrio, M., Castedo, F., Ruíz, A. D., Álvarez, M.F., Álvarez, J. G. y Rojo, A. (2003). Dendrometría. Madrid: Paraninfo.

Farooq, M., Wahid, A., Kobayashi, N., Fujita, D. y Basra, S. M. A. (2009). Plant drought stress: effects, mechanisms and management. Agronomy for Sustainable Development, 29(1), 185-212. https://doi.org/10.1051/agro:2008021

Farrell, C., Szota, C. y Arndt, S. K. (2015). Urban Plantings: 'Living Laboratories' for Climate Change Response. Trends in Plant Science, 20(10), 597-599. https://doi.org/10.1016/j.tplants.2015.08.006

Ferrini, F. y Baietto, M. (2007). Effect of compost-amended backfill and paved surface on leaf parameters and physiology of Norway maple (Acer planatoides L.). Arboriculture \& Urban Forestry, 33(6), 386-391.

Freschet, G. T., Cornelissen, J. H. C., van Logtestijn, R. S. P. y Aerts, R. (2010). Evidence of the 'plant economics spectrum' in a subarctic flora. Journal of Ecology, 98(2), 362-373.

https://doi.org/10.1111/j.1365-2745.2009.01615.x

Godefroid, S. y Koedam, N. (2007). Urban plant species patterns are highly driven by density and function of built-up areas. Landscape Ecology, 22(8), 1227-1239. https://doi.org/10.1007/s10980-007-9102-x

Gómez-Baggethun, E. y Barton, D. N. (2013). Classifying and valuing ecosystem services for urban planning. Ecological Economics, 86, 235-245. https://doi.org/10.1016/j.ecolecon.2012.08.019

Goodness, J., Andersson, E., Anderson, P. M. y Elmqvist, T. (2016). Exploring the links between functional traits and cultural ecosystem services to enhance urban ecosystem management. Ecological Indicators, 70, 597-605.

https://doi.org/10.1016/j.ecolind.2016.02.031

Grabosky, J. y Gilman, E. (2004). Measurement and prediction of tree growth reduction from tree planting space design in established parking lots. Journal of Arboriculture, 30(3), 154-164. 
Grote, R., Samson, R., Alonso, R., Amorim, J. H., Cariñanos, P., Churkina, G., Fares, S., Thiec, D. L., Niinemets, Ü., Mikkelsen, T.N., Paoletti, E., Tiwary, A. y Calfapietra, C. (2016). Functional traits of urban trees: air pollution mitigation potential. Frontiers in Ecology \& the Environment, 14(10), 543-550. https://doi.org/10.1002/fee.1426

Jardín Botánico José Celestino Mutis y Departamento Administrativo Nacional de Estadística (2006). Manual del censista y auxiliar censo del árbol urbano de Bogotá D.C. Bogotá: Jardín Botánico José Celestino Mutis.

Just, M. G., Frank, S. D. y Dale, A. G. (2018). Impervious surface thresholds for urban tree site selection. Urban Forestry \& Urban Greening, 34, 141-146. https://doi.org/10.1016/j.ufug.2018.06.008

Kalusová, V., Čeplová, N. y Lososová, Z. (2017). Which traits influence the frequency of plant species occurrence in urban habitat types? Urban Ecosystems, 20(1), 65-75.

https://doi.org/10.1007/s11252-016-0588-3

Kay, M. y Wobbrock, J. (2019). ARTool: Aligned Rank Transform for Nonparametric Factorial Anovas. R package version 0.10.6.

Konarska, J., Uddling, J., Holmer, B., Lutz, M., Lindberg, F., Pleijel, H. y Thorsson, S. (2016). Transpiration of urban trees and its cooling effect in a high latitude city. International Journal of Biometeorology, 60(1), 159-172.

https://doi.org/10.1007/s00484-015-1014-x

Lavorel, S., Grigulis, K., Lamarque, P., Colace, M. P., Garden, D., Girel, J., Pellet, G. y Douzet, R. (2011). Using plant functional traits to understand the landscape distribution of multiple ecosystem services. Journal of Ecology, 99(1), 135-14. https://doi.org/10.1111/j.1365-2745.2010.01753.x

Lenth, R. (2019). Estimated Marginal Means, aka Least-Squares Means. R package version 1.4.2.

Lovell, S. T. y Taylor, J. R. (2013). Supplying urban ecosystem services through multifunctional green infrastructure in the United States. Landscape Ecology, 28, 1447-1463.

https://doi.org/10.1007/s10980-013-9912-y
McClung, T. e Ibáñez, I. (2018). Quantifying the synergistic effects of impervious surface and drought on radial tree growth. Urban Ecosystems, 21, 147-155. https://doi.org/10.1007/s11252-017-0699-5

Moreno-Barreto, E. y Rubiano, K. (2018). Aproximación al uso de rasgos funcionales y gradientes ambientales para seis especies del arbolado urbano de la ciudad de Bogotá D.C. Bogotá: Jardín Botánico José Celestino Mutis (no publicado).

Mullaney, J., Lucke, T. y Trueman, S. J. (2015). A review of benefits and challenges in growing street trees in paved urban environments. Landscape \& Urban Planning, 134, 157-166.

https://doi.org/10.1016/j.landurbplan.2014.10.013

Mullaney, J., Trueman, S. J., Lucke, T. y Bai, S. H. (2015). The effect of permeable pavements with an underlying base layer on the ecophysiological status of urban trees. Urban Forestry \& Urban Greening, 14(3), 686-693.

https://doi.org/10.1016/j.ufug.2015.06.008

Núñez-Flórez, R., Pérez-Gómez, R. y Fernández-Méndez, F. (2019). Functional diversity criteria for selecting urban trees. Urban Forestry \& Urban Greening, 28, 251-266. https://doi.org/10.1016/j. ufug.2019.01.005

Ocampo, V. (2019). Factors affecting the biodiversity of Heteroptera on trees in urban green areas of Bogotá, Colombia (Tesis de maestría). Universitat de Barcelona, Barcelona, España.

Ordóñez, J. C., Van Bodegom, P. M., Witte, J. P.M., Wright, I. J. Reich, P. B. y Aerts, R. (2009). A global study of relationships between leaf traits, climate and soil measures of nutrient fertility. Global Ecology and Biogeography, 18(2), 137-149.

https://doi.org/10.1111/j.1466-8238.2008.00441.x

Ow, L.F. y Ghosh, S. (2017). Growth of street trees in urban ecosystems: Structural cells and structural soil. Journal of Urban Ecology, 3(1), 1-7. https://doi.org/10.1093/jue/jux017

Pataki, D. E., McCarthy, H. R., Gillespie, T., Jenerette, G. D. y Pincetl, S. (2013). A trait-based ecology of the Los Angeles urban forest. Ecosphere, 4(6), 1-20. https://doi.org/10.1890/es13-00017.1 
Pérez-Harguindeguy, N., Díaz, S., Garnier, E., Lavorel, S., Poorter, H., y Jaureguiberry, P., Bret-Harte, M. S. S., Cornwell, W. K. K., Craine, J. M. M., Gurvich, D. E. E., Urcelay, C., Veneklaas, E. J. J., Reich, P. B. B., Poorter, L., Wright, I. J. J., Ray, P., Enrico, L., Pausas, J. G., de Vos, A. C., Buchmann, N., Funes, G., Quétier, F., Hodgson, J. G., Thompson, K., Morgan, H. D., ter Steege, H., van der Heijden, M. G. A., Sack, L., Blonder, B., Poschlod, P., Vaieretti, M. V., Conti, G., Staver, A. C., Aquino, S. y Cornelissen, J. H. C. (2013). New handbook for standardized measurement of plant functional traits worldwide. Australian Journal of Botany, 61(3), 167-234.

Pretzsch, H., Biber, P., Uhl, E., Dahlhausen, J., Rötzer, T., Caldentey, J., Koike, T., van Con, T., Chavanne, A., Seifert, T., du Toit, B., Farnden, C. y Pauleit, S. (2015). Crown size and growing space requirement of common tree species in urban centres, parks, and forests. Urban Forests \& Urban Greening, 14(3), 466-479.

https://doi.org/10.1016/j.ufug.2015.04.006

R Core Team (2019). R: A Language and Environment for Statistical Computing. Vienna: R Foundation for Statistical Computing.

https://www.R-project.org

Salgado-Negret, B. (ed.) (2015). La ecología funcional como aproximación al estudio, manejo y conservación de la biodiversidad: protocolos y aplicaciones. Bogotá: Instituto de Investigación de Recursos Biológicos Alexander von Humboldt.

Sand, E., Konarska, J., Howe, A. W., Andersson-Sköld, Y., Moldan, F., Pleijel, H. y Uddling, J. (2018). Effects of ground surface permeability on the growth of urban linden trees. Urban Ecosystems, 21, 691-696. https://doi.org/10.1007/s11252-018-0750-1

Sanders, J. R. y Grabosky, J. (2014). 20 years later: Does reduced soil area change overall tree growth? Urban Forestry \& Urban Greening, 13(2), 295-303. https://doi.org/10.1016/j.ufug.2013.12.006

Sanders, J. R., Grabosky, J. y Cowie, P. (2013). Establishing maximum size expectations for urban trees with regard to designed space. Arboriculture \& Urban Forestry, 39(2), 68-73.
Schneider, C. A., Rasband, W. S. y Eliceiri, K. W. (2012). NIH Image to Image): 25 years of image analysis. Nature Methods, 9(7), 671. https://doi.org/10.1038/nmeth.2089

Schwarz, N., Moretti, M., Bugalho, M. N., Davies, Z. G., Haase, D., Hack, J., Hof, A., Melero, Y., Pett, T. J. y Knapp, S. (2017). Understanding biodiversity-ecosystem service relationships in urban areas: A comprehensive literature review. Ecosystem Services, 27, 161-171.

https://doi.org/10.1016/j.ecoser.2017.08.014

Simpson, W. (1993). Specific Gravity, Moisture Content, and Density Relationship for Wood. U.S. Madison, EE. UU.: Department of Agriculture, Forest Service, Forest Products Laboratory.

Song, Y., Li, F., Wang, X., Xu, C., Zhang, J., Liu, X. Y Zhang, H. (2015). The effects of urban impervious surfaces on eco-physiological characteristics of Ginkgo biloba: A case study from Beijing, China. Urban Forestry \& Urban Greening, 14(1), 1102-1109.

https://doi.org/10.1016/j.ufug.2015.10.008

Thompson, K. y McCarthy, M. A. (2008). Traits of British alien and native urban plants. Journal of Ecology, 96(5), 853-859.

https://doi.org/10.1111/j.1365-2745.2008.01383.x

Tovar-Corzo, G. (2007). Manejo del arbolado urbano en Bogotá. Territorios, 16-17, 149-174.

Vallet, J., Daniel, H., Beaujouan, V., Rozé, F. y Pavoine, S. (2010). Using biological traits to assess how urbanization filters plant species of small woodlands. Applied Vegetation Science, 13(4), 412.424. https://doi.org/10.1111/j.1654-109x.2010.01087.x Varis, O. (2007). Megacities, development and water. Water Resources Development, 22(2), 199-225. https://doi.org/10.1080/07900620600648399

Violle, C., Navas, M. L., Vile, D., Kazakou, E., Fortunel, C., Hummel, I. y Garnier, E. (2007). Let the concept of trait be functional! Oikos, 116(5), 882-892. https://doi.org/10.1111/j.0030-1299.2007.15559.x

Viswanathan, B., Volder, A., Watson, W. T. y Aitkenhead-Peterson, J. A. (2011). Impervious and pervious pavements increase soil $\mathrm{CO} 2$ concentrations 
and reduce root production of American sweetgum (Liquidambar styraciflua). Urban Forestry \& Urban Greening, 10(2), 133-139.

https://doi.org/10.1016/j.ufug.2011.01.001

Wang, X., Wang, X., Su, Y. y Zhang, H. (2019). Land pavement depresses photosynthesis in urban trees especially under drought stress. Science of the Total Environment, 653, 120-130.

https://doi.org/10.1016/j.scitotenv.2018.10.281

Williams, N. S. G., Schwartz, M. W., Vesk, P. A., McCarthy, M. A., Hahs, A. K., Clemants, S. E., Corlett, R. T., Duncan, R. P., Norton, B. A., Thompson, K. y McDonnell, M. J. (2009). A conceptual framework for predicting the effects of urban environments on floras. Journal of Ecology, 97(1), 4-9.

https://doi.org/10.1111/j.1365-2745.2008.01460.x

Wobbrock, J. O., Findlater, L., Gergle, D. y Higgins, J. J. (2011). The Aligned Rank Transform for nonparametric factorial analyses using only Anova procedure. En Proceedings of the SIGCHI Conference on Human Factors in Computing Systems (pp. 143146). Nueva York: ACM Press. https://doi.org/10.1145/1978942.1978963

Wright, I. J., Reich, P. B., Westoby, M., Ackerly, D. D., Baruch, Z., Bongers, F., Cavender-Bares, J., Chapin, T., Cornelissen, J. H. C., Diemer, M., Flexas, J., Garnier, E., Groom, P. K., Gulias, J., Hikosaka, K.,
Lamont, B. B., Lee, T., Lee, W., Lusk, C., Midgley, J. J., Navas, M. L., Niinemets, Ü., Oleksyn, J., Osada, H., Poorter, H., Pool, P., Prior, L., Pyankov, V. I. Roumet, C., Thomas, S. C., Tjoelker, M. G., Veneklaas, E. J. y Villar, R. (2004). The worldwide leaf economics spectrum. Nature, 428, 821-827.

https://doi.org/10.1038/nature02403

Xu, F., Guo, W., Xu, W., Wei, Y. y Wang, R. (2009). Leaf morphology correlates with water and light availability: what consequences for simple and compound leaves? Progress in Natural Science, 19, 1789-1798.

https://doi.org/10.1016/j.pnsc.2009.10.001

Yan, Z., Teng, M., He, W., Liu, A., Li, Y. y Wang, P. (2019). Impervious surface area is a key predictor for urban plant diversity in a city undergone rapid urbanization. Science of the Total Environment, 650, 335-342.

https://doi.org/10.1016/j.scitotenv.2018.09.025

Yu, K., Van Geel, M., Ceulemans, T., Geerts, W., Ramos, M. M., Sousa, N., Castro, P. M. L., Kastendeuch, P., Najjar, G., Ameglio, T., Ngao, J., Saudreau, M., Honnay, O. y Somers, B. (2018). Foliar optical traits indicate that sealed planting conditions negatively affect urban tree health. Ecological Indicators, 95, 895-906. https://doi.org/10.1016/j.ecolind.2018.08.047

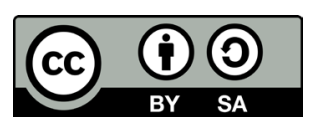

Colombia Forestal •ISSN 0120-0739• e-ISSN 2256-201X • Bogotá-Colombia • Vol. 23 No. 2 • Julio-diciembre de $2020 \bullet$ pp. 5-19. 